\title{
Is radiographic lumbar spondylolisthesis associated with occupational exposures? Findings from a nested case control study within the Wakayama spine study
}

Yuyu Ishimoto ${ }^{1,2,3,4^{*}}$, Cyrus Cooper ${ }^{1,5}$, Georgia Ntani ${ }^{1,5}$, Hiroshi Yamada ${ }^{2}$, Hiroshi Hashizume ${ }^{2}$, Keiji Nagata ${ }^{2}$ Shigeyuki Muraki ${ }^{4}$, Sakae Tanaka ${ }^{6}$, Munehito Yoshida ${ }^{2}$, Noriko Yoshimura ${ }^{4}$ and Karen Walker-Bone ${ }^{1,5}$

\begin{abstract}
Background: To explore the relationship between radiographic spondylolisthesis and occupational factors in a case-control study nested within the Wakayama Spine Study (WSS).

Methods: The WSS is a cross-sectional observational study amongst Japanese adults. All participants completed a lifetime occupational history and underwent X-rays of the lumbar spine (L1-S1) according to a pre-defined protocol. One trained surgeon graded the presence of a spondylolisthesis based upon $\geq 5 \%$ anterior or posterior slip at one or more levels. Cases, with lumbar spondylolisthesis, were compared with controls without, for their principal occupation and occupational exposures.

Results: In total, data were available for 722 adults (245 men and 477 women), mean age 70.1 (range 53-93) years. According to the pre-defined radiographic criteria, 117 were defined with spondylolisthesis (cases), leaving 605 controls. Cases were not significantly different from controls for age, gender, BMl, smoking or alcohol intake. However, cases were more than twice as likely to report occupational driving $\geq 4$ h/day (OR 2.39, 95\% Cl 1.08-5.27) after adjustment for age, gender and BMI. Additionally, after stratification by age using 75 years as a cut-point, cases were more than 3-fold more likely to report having worked in the agricultural/ fishing industries (OR 3.47, 95\% Cl 1.29-9.29) among those aged $<75$ years. A reduced risk of being a case was associated with climbing slopes/steps and walking.

Conclusions: A history of occupational driving and working in the agricultural/fishing industry were associated with radiographic spondylolisthesis in this cross-sectional population study. This finding requires further evaluation in longitudinal studies.
\end{abstract}

Keywords: Spondylolisthesis, Occupation, Agricultural /driving

\section{Background}

Spondylolisthesis describes the anterior or posterior migration, or slip, of one vertebra in relation to the next caudal vertebra. The first-line investigation for spondylolisthesis is lumbar spine radiography, studies of which have shown that the characteristic changes are rare

\footnotetext{
* Correspondence: yuyu.ishimoto@hotmail.co.jp

${ }^{1}$ MRC Lifecourse Epidemiology Unit, Southampton General Hospital, Southampton, Hampshire, UK

${ }^{2}$ Orthopedic surgery, Wakayama Medical University, Wakayama city,

Wakayama prefecture, Japan

Full list of author information is available at the end of the article
}

below aged 50 years but that the prevalence increases sharply with age affecting as many as $15 \%$ of men and more than $50 \%$ of women aged $66-70$ years [1-3]. It has been demonstrated that the severity of radiographic changes is associated with the risk of symptoms of pain and disability [1]. Disability arises from any combination of low back pain, neurogenic claudication and radiculopathy, associated with disc degeneration, compromise of the canal and central spinal stenosis and/or foraminal narrowing [4]. An incidence rate as high as $4.1 \%$ has been estimated in the general population [5]. For the

(c) The Author(s). 2019 Open Access This article is distributed under the terms of the Creative Commons Attribution 4.0 International License (http://creativecommons.org/licenses/by/4.0/), which permits unrestricted use, distribution, and reproduction in any medium, provided you give appropriate credit to the original author(s) and the source, provide a link to the Creative Commons license, and indicate if changes were made. The Creative Commons Public Domain Dedication waiver (http://creativecommons.org/publicdomain/zero/1.0/) applies to the data made available in this article, unless otherwise stated. 
most severe symptoms, surgical management is indicated [6] and rates of surgery for this condition are increasing [7]. Given steady increases in healthy life expectancy, the burden of this condition is likely to increase.

From a public health perspective, understanding the epidemiology and risk factors for this condition could enable us to understand factors which determine longitudinal progression and facilitate the development of preventive strategies to reduce the impact of this disabling condition. To date, age, female gender, ethnicity, hormonal factors (menopause, pregnancy), height, BMI, pelvic and spinal structural factors have been implicated $[1,8]$ but most of these factors are not, unfortunately, modifiable. Sports and sporting activities, particularly gymnastics, have been associated in some studies [9-13]. Therefore, It has been suggested that more epidemiological studies to define potentially modifiable environmental factors are indicated [14].

There has been evidence for some time that physical workplace exposures are associated with an increased risk of low back pain [15] and degenerative low back pain in particular $[16,17]$ and therefore, occupational factors could be implicated in spondylolisthesis but there has been limited research to date. The one published study among helicopter pilots described an increased risk of lytic spondylolisthesis [18] and a study among taxi drivers reported an increased risk [14]. More recently, Mariconda and colleagues reported an increased association with self-reported "heavy workload" but not with heaviness of self-reported loads lifted at work or occupational driving and reported a reduced risk associated with "lifetime occupational exposure" and prolonged occupational standing [19].

Therefore, we carried out a case-control study to investigate the association of occupation and occupational activities with radiographic spondylolisthesis among a population sample of older adults in the Wakayama Spine Study (WSS).

\section{Methods}

\section{Hypothesis}

That occupation and occupational factors are associated with an increased risk of radiographic spondylolisthesis.

\section{Participants}

Under the approval of our institutional review board, the present study, entitled the Wakayama Spine Study (WSS), was performed with a sub-cohort of the Research on Osteoarthritis/Osteoporosis Against Disability (ROAD) study. The ROAD study was initiated as a nationwide, prospective study of bone and joint diseases in population-based cohorts. A detailed profile of the ROAD cohort has been previously reported [20, 21]. Therefore, in brief, the ROAD study included 3040 inhabitants (1061 men and 1979 women) aged 23-95 years recruited from resident registries in three communities. ROAD included an urban community, Itabashi-ku, but the WSS, which for convenience was the sampling frame for the current study, only included participants from the two rural communities near Wakayama: Hidakagawa and Taiji. Hidakagawacho, is a mountainous community located in the center of Wakayama, which had a population of $11,300 / 330 \mathrm{~km}^{2}$ with $29 \%$ of jobs in primary industries (agriculture, forestry, fishing and mining), $24 \%$ in the secondary industries (manufacturing and construction), and $47 \%$ of jobs in the service industry. Taiji-cho, is a seacoast community located south of Wakayama, with a population of 3500/6 $\mathrm{km}^{2}$. In comparison with the above, $13 \%$ of the Taiji population work in the primary industries, $18 \%$ in the secondary industries and $69 \%$ work in the service industry. The ROAD study team made a second visit to Hidakagawa and Taiji between 2008 and 2010. Of the inhabitants who participated in this second visit, 1063 volunteers were recruited for MRI. Fifty-two of these declined to attend the examination, and the remaining 1011 were registered in the Wakayama Spine Study. All participants provided their written, informed consent for the MRI examination. Participants who had sensitive implanted devices (such as a pacemaker) or other disqualifiers were excluded. In total, 977 participants underwent lumbar spine MRI. Ten participants who had undergone a previous lumbar operation were excluded, and 29 participants who were younger than 40 years were excluded. All participants in the WSS were invited to complete an interviewer-administered questionnaire which included 400 questions about demographic factors, lifestyle factors, occupation, and occupational exposures and underwent lumbar spine radiographs and anthropometric measurements. Everybody was eligible to participate, regardless of age, gender and symptoms at baseline, providing that they could give written, informed consent and were able to complete the questionnaire and undergo spinal radiography (pregnant women were excluded). Complete radiographic and occupational data were available for 722 participants ( 245 males, 477 females), mean age 70.9 years, range: $53-93$ years.

The study was approved by the ethics committees of the University of Tokyo and the Tokyo Metropolitan Institute of Gerontology.

\section{Occupation and occupational activities}

A lifetime occupational history was collected alongside details of 7 types of specific work exposures: sitting on a chair for $\geq 2 \mathrm{~h} /$ day; standing for $\geq 2 \mathrm{~h} /$ day; kneeling for $\geq 1 \mathrm{~h} /$ day; squatting for $\geq 1 \mathrm{~h} /$ day; driving for $\geq 4 \mathrm{~h} /$ day; walking $\geq 3 \mathrm{~km} /$ day; going up and down stairs $\geq 30$ floors/day; climbing up slopes or steps for $\geq 1 \mathrm{~h} /$ day and; lifting loads weighing $\geq 10 \mathrm{~kg}$ at least once a week. For 
the current study, the information on occupational title and exposures was derived from the respondent's principal occupation (that in which the participant had worked for the longest duration). For comparison, occupations were grouped according to the nature of work as follows: Clerical/technical; agricultural / fishermen; factory/construction; clinical / housekeepers / shop workers / hairdressers / dressmakers; teachers and "other" (for all remaining types of work).

\section{Assessment of lumbar spondylolisthesis}

Lumbar spine radiographs were performed according to a standardised protocol to include the intervertebral levels from L1-L2 to L5-S1. Anteroposterior and lateral radiographs of the lumbar spine were acquired with patients in a standing position. The radiographs were all read without the knowledge of participant symptoms, occupational exposures or function by one experienced orthopaedic surgeon (YI). In line with other epidemiological studies of radiographic spondylolisthesis [22-24], the \%slip was calculated as the distance of sagittal translation between adjacent vertebral endplates. A patient was defined with spondylolisthesis if they had a slip $\geq 5 \%$ anteriorly or posteriorly at any lumbar level on the lateral views [22-24].

\section{Statistical analysis}

Participants' demographic and lifestyle characteristics were summarized using means (SDs) where normally distributed and medians (inter-quartile ranges, IQRs) when not and counts (\%) separately for those with spondylolisthesis (cases) and those without (controls). Differences in categorical and continuous characteristics between cases and controls were assessed using chisquared and $t$-tests, respectively. The effects of type of occupation (using clerical/technical experts as a baseline category), and occupational activities on spondylolisthesis were assessed using logistic regression modelling, before and after adjusting for demographic characteristics, and were summarized by odds ratios (ORs) and 95\% confidence intervals (CIs).

As the main focus of this study was to explore the association between occupational factors and spondylolisthesis, and many of the older participants had stopped working as much as 20-30 years prior to their X-ray, we repeated the analyses separately for those $<75$ and $\geq 75$ years of age, allowing a decade after retirement. Statistical analyses were performed using Stata V.12.1 (StataCorp, College Station, Texas, USA).

\section{Results}

Firstly, the inter- and intra-observer reliability of the radiographic assessment of spondylolisthesis was assessed in two sub-studies. For intra-observer reliability, the orthopaedic surgeon re-assessed a random sample of 50 lumbar spine radiographs a month later, blinded to his original assessment and in a different order. A kappa of 0.85 was obtained for intra-observer agreement. Secondly, in a different sample of 50 randomly-selected radiographs, another experienced observer (SM) assessed the \% slip at levels L3-5 for 150 levels of radiographs and obtained similarly excellent levels of inter-observer agreement (kappa $=0.83$ ) for the presence/absence of a slip.

In total, 117 (16.2\%) of participants were defined with spondylolisthesis (cases) and the remaining 605 individuals as controls (Table 1). There were no differences between cases and controls in terms of sex, age, BMI, usual walking speed (as a proxy for physical function), smoking and alcohol.

Table 2 shows the associations between occupational group and occupational activities amongst cases as compared with controls. In the unadjusted analyses (Model 1) no association was found between spondylolisthesis and working in any occupational group (agricultural/fishermen; factory/construction; clinical / housekeepers / shop workers / hairdressers / dressmakers; teachers or "other"), as compared with the referent clerical/technical workers.

When considering occupational exposures, cases were more likely to report exposure to occupational driving $\geq 4 \mathrm{~h}$ /day than controls (OR 2.17, 95\% CI 1.01-4.64). After adjustment for age and sex (Model 2), the association was if anything stronger (OR 2.49, CI 1.13-5.49) and was robust even after full adjustment for age, sex and BMI (OR 2.39, CI 1.08-5.27) (Model 3). We found no other occupational exposures to be associated, with or without adjustment.

Table 3 presents the stratified analysis by age using 75 years as a cut-point. Amongst younger participants (aged $<75$ years), an increased association was found amongst cases as compared with controls for working in agricultural/fishing industry as compared with clerical/technical work (OR 3.41, CI 1.32-8.81) (Model 1) and this was robust to adjustment for age, gender and BMI (OR 3.47, CI 1.29-9.29) (Model 2) (Fig. 1). Once again, we found no other association with the other types of occupation (factory/construction; clinical / housekeepers / shop workers / hairdressers / dressmakers; teachers or "other"). No exposures were found significantly associated amongst those aged $\geq 75$ years.

Exploring occupational exposures stratified by age, we found a reduced odds ratio for being a case amongst those reporting exposure to climbing up slopes or steps for $\geq 1 \mathrm{~h}$ /day in those aged $<75$ years with and without full adjustment (Model 1: OR 0.38, CI 0.17-0.88; Model 2: OR 0.36, 0.15-0.82). Amongst the older participants (aged $\geq 75$ years) cases were more likely to report sitting on a chair for $\geq 2 \mathrm{~h} /$ day in the unadjusted (Model 1) (OR 1.87, CI 1.03-3.40) and adjusted models (OR 1.95, CI 
Table 1 Characteristics of cases with spondylolisthesis and controls

\begin{tabular}{llll}
\hline & Cases $(N=117)$ & Controls $(N=605)$ & $p$-value \\
\hline Sex & & & \\
$\quad$ Males & $35(29.9 \%)$ & $210(34.7 \%)$ & 0.316 \\
Age (mean (SD)) & $71.5(9.9)$ & $70.8(9.9)$ & 0.505 \\
BMI (mean (SD)) & $23.7(3.5)$ & $23.1(3.4)$ & 0.069 \\
Usual walking speed (median (IQR)) & $5(5-6)$ & $5(5-6)$ & 0.759 \\
Smoking & & & 0.823 \\
$\quad$ No & $108(92.3 \%)$ & & $0.932(91.9 \%)$ \\
Alcohol & & $432(71.4 \%)$ & \\
$\quad$ No & $84(71.8 \%)$ & & \\
\hline
\end{tabular}

1.06-3.57) (Model 2). Additionally, reported walking $\geq 3$ $\mathrm{km} /$ day at work conveyed a reduced risk (OR 0.53, CI $0.29-0.98)$ in the unadjusted analyses but this association became non-significant after full adjustment (Model 2).

\section{Discussion}

The results of this study suggest that cases reported a more than doubling of the risk of exposure to occupational driving $\geq 4$ h/day (adjusted OR 2.39, 95\% CI 1.085.27) in unstratified analyses. Stratification of the analyses by age provided additional insight as, amongst those aged $<75$ years, we found that cases were more than 3-fold likely to report exposure to heavy manual work in the agricultural/fishing industries (adjusted OR 3.47, 95\%CI 1.29-9.29). Spondylolisthesis cases were more likely to report exposure to driving $\geq 4 \mathrm{~h} /$ day in the stratified analyses but these did not attain statistical significance. However, spondylolisthesis was associated with an almost doubling of risk of reporting sedentary work $\geq 2 \mathrm{~h}$ /day amongst those aged $\geq 75$ years (OR 1.95, 95\% CI 1.06-3.57). Additionally, we found that there was a negative association with spondylolisthesis (OR 0.36, 95\% CI 0.15-0.82) amongst those aged $<75$ years who reported that they climbed up stairs and slopes $\geq 1$ $\mathrm{h} /$ day and a similar negative association was seen with walking $\geq 3 \mathrm{~km} /$ day amongst those aged $\geq 75$ years (OR $0.55,95 \%$ CI $0.29-1.02$ ).

Table 2 Comparison of associations with occupation and occupational activities of cases of spondylolisthesis with controls

\begin{tabular}{|c|c|c|c|c|c|c|c|c|c|c|c|c|}
\hline & \multirow{2}{*}{$\begin{array}{l}\text { Total } \\
\mathrm{N}\end{array}$} & \multirow[t]{2}{*}{ Cases } & \multirow[t]{2}{*}{ Controls } & \multicolumn{3}{|c|}{ Model 1} & \multicolumn{3}{|c|}{ Model 2} & \multicolumn{3}{|c|}{ Model 3} \\
\hline & & & & $\overline{\mathrm{OR}}$ & $95 \% \mathrm{Cls}$ & $P$ & $\overline{\mathrm{OR}}$ & $95 \% \mathrm{Cls}$ & $P$ & $\overline{O R}$ & $95 \% \mathrm{Cls}$ & $P$ \\
\hline \multicolumn{13}{|l|}{ Occupational group } \\
\hline Clerical / technical experts & 197 & 29 & 168 & 1.0 & & & 1.0 & & & 1.0 & & \\
\hline Agricultural / Fishermen & 105 & 16 & 89 & 1.04 & $(0.54,2.02)$ & 0.9 & 1.01 & $(0.50,2.01)$ & 0.99 & 0.99 & $(0.50,1.98)$ & 0.98 \\
\hline Factory/construction & 48 & 10 & 38 & 1.52 & $(0.68,3.39)$ & 0.3 & 1.49 & $(0.66,3.34)$ & 0.33 & 1.46 & $(0.65,3.27)$ & 0.36 \\
\hline $\begin{array}{l}\text { Clinical / Housekeepers / Shop workers / } \\
\text { Hairdressers / Dressmakers }\end{array}$ & 207 & 39 & 168 & 1.34 & $(0.79,2.28)$ & 0.27 & 1.27 & $(0.74,2.19)$ & 0.39 & 1.24 & $(0.72,2.14)$ & 0.45 \\
\hline Teachers & 51 & 6 & 45 & 0.77 & $(0.30,1.97)$ & 0.59 & 0.77 & $(0.30,1.99)$ & 0.59 & 0.78 & $(0.30,2.00)$ & 0.6 \\
\hline Other/NA & 114 & 17 & 97 & 1.02 & $(0.53,1.94)$ & 0.96 & 0.99 & $(0.51,1.89)$ & 0.97 & 0.97 & $(0.50,1.86)$ & 0.92 \\
\hline \multicolumn{13}{|l|}{ Occupational activities } \\
\hline Sitting on a chair $\geq 2 \mathrm{~h} /$ day & 347 & 60 & 287 & 1.17 & $(0.78,1.73)$ & 0.45 & 1.21 & $(0.81,1.81)$ & 0.35 & 1.21 & $(0.81,1.81)$ & 0.36 \\
\hline Standing $\geq 2$ h/day & 574 & 87 & 487 & 0.7 & $(0.44,1.11)$ & 0.13 & 0.68 & $(0.43,1.09)$ & 0.11 & 0.67 & $(0.42,1.08)$ & 0.1 \\
\hline Kneeling $\geq 1$ h/day & 115 & 21 & 94 & 1.19 & $(0.71,2.00)$ & 0.51 & 1.16 & $(0.69,1.97)$ & 0.57 & 1.13 & $(0.67,1.91)$ & 0.65 \\
\hline Squatting $\geq 1 \mathrm{~h} /$ day & 159 & 26 & 133 & 1.01 & $(0.63,1.63)$ & 0.95 & 1 & $(0.61,1.61)$ & 0.99 & 0.97 & $(0.60,1.58)$ & 0.91 \\
\hline Driving $\geq 4 \mathrm{~h} /$ day & 35 & 10 & 25 & 2.17 & $(1.01,4.64)$ & 0.05 & 2.49 & $(1.13,5.49)$ & 0.02 & 2.39 & $(1.08,5.27)$ & 0.03 \\
\hline Walking $\geq 3$ km/day & 238 & 35 & 203 & 0.85 & $(0.55,1.30)$ & 0.44 & 0.82 & $(0.52,1.28)$ & 0.37 & 0.81 & $(0.52,1.28)$ & 0.37 \\
\hline Going up/down stairs $\geq 30$ floors/day & 169 & 20 & 149 & 0.63 & $(0.38,1.06)$ & 0.08 & 0.64 & $(0.38,1.07)$ & 0.09 & 0.62 & $(0.37,1.05)$ & 0.07 \\
\hline Climbing up slopes or steps $\geq 1 \mathrm{~h} /$ day & 94 & 11 & 83 & 0.65 & $(0.34,1.27)$ & 0.21 & 0.6 & $(0.30,1.22)$ & 0.16 & 0.63 & $(0.31,1.27)$ & 0.2 \\
\hline Lifting loads of $\geq 10 \mathrm{~kg}$ at least once per week & 327 & 45 & 282 & 0.72 & $(0.48,1.07)$ & 0.11 & 0.73 & $(0.48,1.10)$ & 0.13 & 0.72 & $(0.47,1.09)$ & 0.12 \\
\hline
\end{tabular}


Table 3 Comparison of associations of cases of spondylolisthesis with controls by occupational group and occupational activities stratified by age

\begin{tabular}{|c|c|c|c|c|c|c|c|c|c|c|c|c|}
\hline & \multicolumn{6}{|c|}{$<75$ years } & \multicolumn{6}{|c|}{$>=75$ years } \\
\hline & \multicolumn{3}{|c|}{ Model 1} & \multicolumn{3}{|c|}{ Model 2} & \multicolumn{3}{|c|}{ Model 1} & \multicolumn{3}{|c|}{ Model 2} \\
\hline & OR & $95 \% \mathrm{Cls}$ & $P$ & OR & $95 \% \mathrm{Cls}$ & $P$ & OR & $95 \% \mathrm{Cls}$ & $P$ & OR & $95 \% \mathrm{Cls}$ & $P$ \\
\hline \multicolumn{13}{|l|}{ Occupational group } \\
\hline Clerical / technical experts & 1.0 & & & 1.0 & & & 1.0 & & & 1.0 & & \\
\hline Agricultural / Fishermen & 3.41 & $(1.32,8.81)$ & 0.01 & 3.47 & $(1.29,9.29)$ & 0.01 & 0.43 & $(0.16,1.16)$ & 0.1 & 0.43 & $(0.16,1.17)$ & 0.1 \\
\hline Factory/construction & 2.92 & $(0.99,8.64)$ & 0.05 & 2.88 & $(0.94,8.78)$ & 0.06 & 0.72 & $(0.21,2.47)$ & 0.6 & 0.71 & $(0.21,2.43)$ & 0.58 \\
\hline $\begin{array}{l}\text { Clinical / Housekeepers / Shop workers / Hairdressers } \\
\text { / Dressmakers }\end{array}$ & 1.44 & $(0.73,2.84)$ & 0.29 & 1.33 & $(0.66,2.69)$ & 0.43 & 1.18 & $(0.51,2.71)$ & 0.71 & 1.07 & $(0.45,2.59)$ & 0.87 \\
\hline Teachers & \multicolumn{12}{|c|}{ No cases } \\
\hline Other/NA & 0.96 & $(0.40,2.28)$ & 0.93 & 0.91 & $(0.38,2.17)$ & 0.83 & 1.05 & $(0.39,2.84)$ & 0.92 & 1.01 & $(0.37,2.76)$ & 0.99 \\
\hline \multicolumn{13}{|l|}{ Occupational activities } \\
\hline Sitting on a chair $\geq 2 \mathrm{~h} /$ day & 0.81 & $(0.48,1.39)$ & 0.45 & 0.83 & $(0.48,1.42)$ & 0.49 & 1.87 & $(1.03,3.40)$ & 0.04 & 1.95 & $(1.06,3.57)$ & 0.03 \\
\hline Standing $\geq 2$ h/day & 0.76 & $(0.42,1.36)$ & 0.35 & 0.73 & $(0.40,1.31)$ & 0.29 & 0.56 & $(0.25,1.22)$ & 0.14 & 0.54 & $(0.24,1.20)$ & 0.13 \\
\hline Kneeling $\geq 1$ h/day & 0.98 & $(0.46,2.11)$ & 0.96 & 0.94 & $(0.43,2.04)$ & 0.87 & 1.41 & $(0.69,2.90)$ & 0.35 & 1.35 & $(0.65,2.79)$ & 0.42 \\
\hline Squatting $\geq 1 \mathrm{~h} /$ day & 1 & $(0.49,2.03)$ & 1 & 0.92 & $(0.44,1.90)$ & 0.82 & 1 & $(0.52,1.93)$ & 0.99 & 0.98 & $(0.51,1.88)$ & 0.94 \\
\hline Driving $\geq 4$ h/day & 2.34 & $(0.79,6.88)$ & 0.12 & 2.33 & $(0.77,7.07)$ & 0.14 & 2 & $(0.68,5.86)$ & 0.21 & 2.6 & $(0.83,8.15)$ & 0.1 \\
\hline Walking $\geq 3$ km/day & 1.32 & $(0.71,2.47)$ & 0.38 & 1.25 & $(0.66,2.35)$ & 0.49 & 0.53 & $(0.29,0.98)$ & 0.04 & 0.55 & $(0.29,1.02)$ & 0.06 \\
\hline Going up/down stairs $\geq 30$ floors/day & 0.38 & $(0.17,0.88)$ & 0.02 & 0.36 & $(0.15,0.82)$ & 0.02 & 0.97 & $(0.49,1.91)$ & 0.92 & 0.99 & $(0.50,1.97)$ & 0.97 \\
\hline Climbing up slopes or steps $\geq 1 \mathrm{~h} /$ day & 0.89 & $(0.19,4.07)$ & 0.88 & 0.98 & $(0.21,4.56)$ & 0.98 & 0.55 & $(0.26,1.18)$ & 0.13 & 0.56 & $(0.25,1.23)$ & 0.15 \\
\hline Lifting loads of $\geq 10 \mathrm{~kg}$ at least once per week & 0.88 & $(0.51,1.53)$ & 0.65 & 0.88 & $(0.50,1.55)$ & 0.65 & 0.55 & $(0.30,1.00)$ & 0.05 & 0.57 & $(0.31,1.04)$ & 0.07 \\
\hline
\end{tabular}

Model 1: Unadjusted OR; Model 2: Adjusted for age, sex, and BMI

The finding that the associations of spondylolisthesis with agricultural/fisherman become attenuated at older ages ( $\geq 75$ years) is not surprising. The retirement age in many Japanese industries is $<65$ years so that, in this older cohort, many participants would have stopped work some years prior to their X-rays. It is clear from epidemiological studies that age, gender and hormonal factors play a role in the pathogenesis in spondylolisthesis and it is likely that the impact of occupational exposures overall will relatively diminish as that of other exposures (leisure-time physical activities) increase as years since retirement increases $[1,8]$.

The results need to be considered alongside some limitations. First, this is a cross-sectional study so that causal attribution cannot be made. Secondly, the participants in this study were sampled from the general population but not at random. We investigated their representativeness by comparing the study population with the general population of Japan for a key risk factor for osteoarthritis, body mass index (BMI). We found that the mean BMI of the participants was not significantly different from that of the general population of Japan (males: 23.71 (3.41) vs. 23.95 (2.64) $\mathrm{kg} / \mathrm{m} 2$; females: $23.06(3.42)$ vs. $23.50(3.69) \mathrm{kg} / \mathrm{m} 2)$. However, the study participants reported a lower prevalence of smoking and alcohol use than that seen in the Japanese general population, suggesting participants might live healthier lifestyles. This may limit the generaliseability of these findings. We also cannot rule out a possible selection bias as volunteers needed to be sufficiently healthy to participate and undergo spinal radiographs and this may have limited the possible involvement of elderly institutionalised adults. As spondylolisthesis is a common cause of impaired mobility in older people and immobility may lead to institutionalisation, this may have created a bias, but if so, the effect would have been to reduce the estimated prevalence of spondylolisthesis. The impact of this on the study results would however, only be biased if we believe that those who were employed in any specific occupation were more likely to be institutionalised than those who had worked in others, which seems unlikely. Occupational exposures were obtained by direct inquiry rather than being inferred from job title. Of course the information is dependent upon recall, but the subjects were unaware of their radiographic findings when they were recalling their occupation and occupational exposures so that a systematic bias is unlikely.

It is a strength of this study that all $\mathrm{X}$-rays were assessed by one highly-trained orthopaedic surgeon (YI). In addition, considerable efforts were made to guarantee the reliability of the readings, including inter-observer and intra-observer studies with a sample of $5 \%$ of the X- 


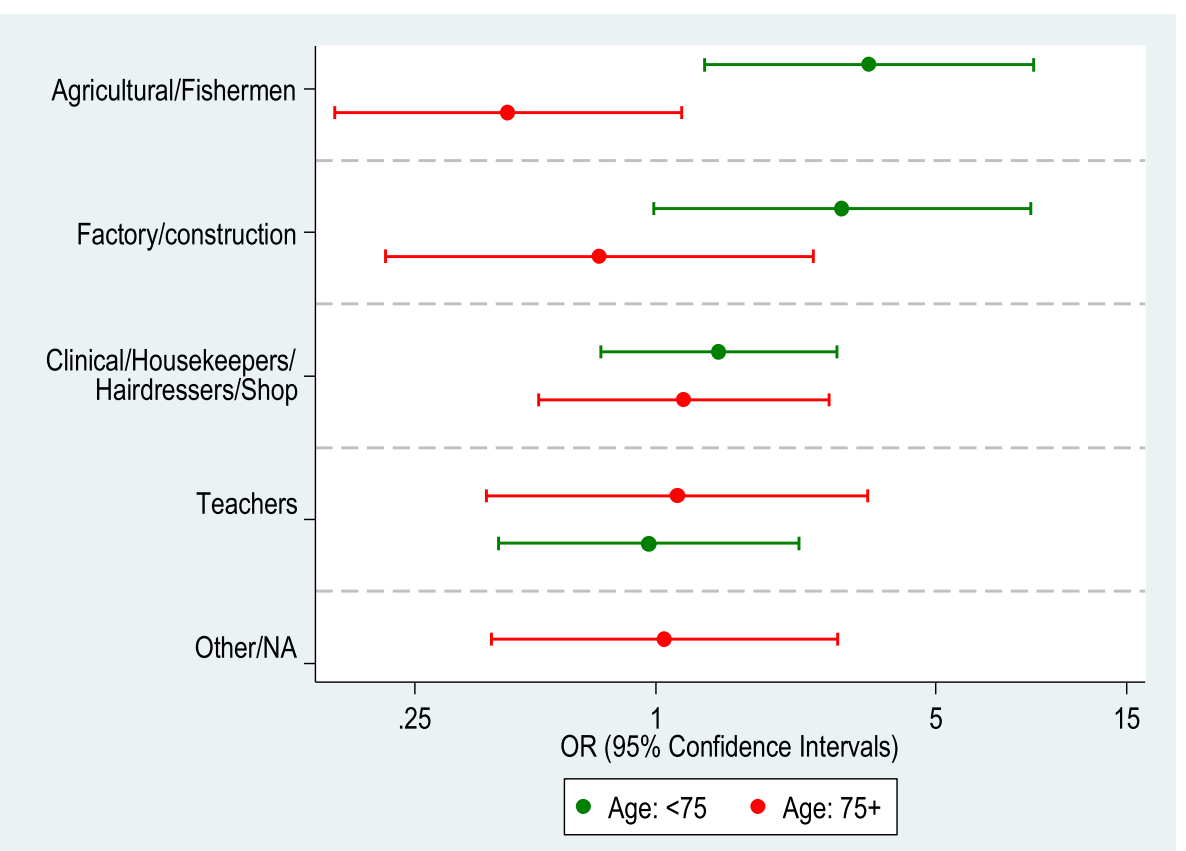

Fig. 1 Comparison of the adjusted associations (OR and 95\% Cls) among cases with spondylolisthesis, as compared with controls without, among people from the different occupational, stratified by age

rays, both of which suggested a very good level of reliability (kappa $>0.8$ in both studies).

There is disagreement in the literature as to how best to classify radiographic spondylolisthesis. Two principal types are proposed: lytic and degenerative. Lytic is associated with a pars defect of the vertebral arch and usually causes slippage at younger ages. Degenerative spondylolisthesis has been variously described but usually defines a slip occurring with an intact vertebral arch with/without associated advanced arthritis changes in the facet joints at the same level [8]. A systematic review of risk factors for spondylolisthesis found that the selection of cases for observational studies has differed widely [8], some studies restricting selection of cases to those with a slip at L4-5 but others including a slip at any level. Some, but not all, studies included anterior and posterior slips and some studies selected only symptomatic spondylolisthesis or surgical cases. Selection based upon symptoms was criticized in the review given the lack of conclusive evidence that spondylolisthesis causes low back pain. Given the current lack of clarity about case definition, we chose a case definition which is inclusive and consistent with that of other researchers [22-24]. In particular, it was not our intention to separate lytic from degenerative types of spondylolisthesis but rather to use this large population sample to investigate whether any type of occupational exposure was associated with the outcome, in order to generate hypotheses about causation and potentially develop preventive strategies. This is particularly important with occupational research where there is already an established tradition of putting in place preventive strategies to protect workers if an increased risk is established, often in the absence of information about mechanism of causation (e.g. asbestos and lung disease).

Low back pain associated with manual occupations has long been reported [25]. Degenerative changes at the cervical and lumbar spine have been described with working outdoors, heavy lifting and whole-body vibration [25-27]. An increased risk of disc degeneration on MRI scans has been reported amongst those who had undertaken the heaviest occupational lifting [28] and highest lifetime cumulative lifting load [29]. Moreover, increased rates of radiographic lumbar spondylosis were found amongst agricultural/forestry/fishery workers [26], and an increased risk of vertebral endplate sclerosis amongst concrete reinforcement workers [14]. In another recent analysis within the Wakayama Spine Study, we have shown an increased association of lumbar spinal stenosis with factory and construction work [submitted to Am J Ind Med]. The current study adds to a growing body of literature suggesting that the risk of spinal structural degenerative changes is increased in some occupations. The different occupational risk profiles warrant further investigation but might be explained by the selective mechanical effects of these exposures.

Spondylolisthesis has been previously reported in association with occupational driving and flying. Froom et al. 
reported a four-fold increased risk of lytic spondylolisthesis amongst helicopter pilots as compared with transport pilots and cadets [17]. Among Taiwanese taxi drivers, longer duration of exposure to driving was associated with an increased risk of spondylolisthesis [18]. Mariconda and colleagues found that, amongst 120 back pain patients with spondylolisthesis, occupational driving was the only factor associated with a greater degree of vertebral slip and other occupational activities (awkward posture, prolonged sitting and prolonged standing) were not [19]. It has been postulated that the mechanism by which driving/flying increase the risk of spondylolisthesis is through exposure to whole-body vibration. Indeed, animal studies found that exposure to continuous quantitative vibration diminished the proteoglycan content in the nucleus pulposus disrupting the integrity of the matrix and increasing the instability of the intervertebral disc [30]. The results of our study support the possibility of an association with occupational driving and of course workers in the agricultural/fishing industries may also be exposed to whole-body vibration in tractors or boats [31, 32].

The evidence about other occupational exposures and spondylolisthesis is conflicting. One study reported an increased risk of symptomatic spondylolisthesis amongst those with a heavy workload and undertaking manual handling of materials [19] but another found no association between risk of degenerative spondylolisthesis and the degree of lifting [33]. A study using the same radiographic definition of spondylolisthesis as in the current study found that, although the prevalence of individuals reporting that their 'longest occupation involved physical labour' was higher among men with a slip (10.2\%) than those without $(8.6 \%)$, the differences were not statistically significant [24]. It is interesting that we found an association with occupational sitting but only amongst older participants after stratification by age. Two other studies reported no association [19, 33] but differed importantly in their methodology: one [19] only included back pain patients attending outpatient clinics and willing to undergo MRI whilst the other [33] recruited > 4000 people to a population cohort but included a wider age range (22-93), so that the participants were on average 10 years younger than in the current study. More research is required to explore the impact of prolonged occupational sitting but, as sedentary work is becoming increasingly common with mechanization, this could be an important risk factor for spondylolisthesis. Importantly, our findings of a negative association with reported climbing flights of stairs or slopes $\geq 1 \mathrm{~h} /$ day and/or walking $\geq 3 \mathrm{~h} /$ day may suggest that, if replicated in longitudinal studies, being active during working hours may be beneficial in the long-term prevention of this condition.

\section{Conclusions}

We have shown that occupational driving and working in the Agricultural/Fishery industries is associated with spondylolisthesis in this cross-sectional study. Our results also point to a possible increased risk from sedentary work and reduced risk from work that requires walking or climbing up/down slopes or steps. These findings need replication in longitudinal studies to confirm cause/effect but could have implications for countries that define and compensate 'industrial injuries'.

\section{Abbreviations}

BMI: Body mass index; Cl: Confidencel interval; MRI: Magnetic resonance imaging; OR: Odds ratio; WSS: Wakayama Spine Study

\section{Acknowledgements}

The authors wish to thank Mrs. Tomoko Takijiri and other members of the Public Office in Hidakagawa Town, and Mrs. Tamako Tsutsumi, Mrs. Kanami Maeda, and other members of the Public Office in Taiji Town, for their assistance in locating and scheduling participants for examinations. The corresponding author had full access to all the data and had the final decision to submit for publication.

\section{Authors' contributions}

All authors worked collectively to develop the protocols and methods described in this paper. YI, SM, KN, HH, HY, ST, MY, and NY were the principal investigators responsible for the fieldwork in the Wakayama Spine Study. GN performed the statistical analysis. YI, CC, and KWB contributed to the analysis and interpretation of results. YI wrote the report. All authors read and approved the final report.

\section{Funding}

Supported by Grants-in-Aid for Scientific Research (B20390182, B23390357, C20591737, C20591774), Young Scientists (A18689031), and Exploratory Research (19659305) from the Japanese Ministry of Education, Culture, Sports, Science and Technology; Grants-in-Aid, H17-Men-eki-009, H18-Choujyu-037, and H20-Choujyu-009 from the Ministry of Health, Labour and Welfare; Research Aid from the Japanese Orthopaedic Association; a grant from the Japanese Orthopaedics and Traumatology Foundation, Inc. (No. 166); and a Grant-in-Aid for Scientific Research, Scientific Research (C22591639) from the Japan Society for the Promotion of Science; and the 2012 Wakayama Medical Award for Young Researchers; and Grant-in-Aid for the Promotion of joint International Research (15KK0316) from Japan Society for the Promotion of Science. The sponsors had no role in study design, data collection, data analysis, data interpretation, or in the writing of the report.

\section{Availability of data and materials}

The authors will provide anonymized data on request as long as researchers are qualified to request these data. Data requests can be made to Wakayama Medical University Ethics Committee at wa-rinri@wakayama-med.ac.jp

\section{Ethics approval and consent to participate}

The study was approved by the ethics committees of the University of Tokyo and the Tokyo Metropolitan Institute of Gerontology. All participants provided their written, informed consent for joining the study and the MRI examination.

\section{Consent for publication}

All participants also provided their written, informed consent for consent for publication.

\section{Competing interests}

Shigeyuki Muraki who is our co-author is a member of the editorial board (Associate Editor) of BMC Musculoskeletal Disorders.

\section{Author details}

${ }^{1}$ MRC Lifecourse Epidemiology Unit, Southampton General Hospital, Southampton, Hampshire, UK. ${ }^{2}$ Orthopedic surgery, Wakayama Medical 
University, Wakayama city, Wakayama prefecture, Japan. ${ }^{3}$ Orthopedic surgery, Kinan Hospital, Tanabe city, Wakayama prefecture 646-8588, Japan.

${ }^{4}$ Department of Preventive Medicine for Locomotive Organ Disorders, 22nd Century Medical \& Research Center, Faculty of Medicine, University of Tokyo, Tokyo, Japan. ${ }^{5}$ Arthritis Research UK/MRC Centre for Musculoskeletal Work and Health, Southampton General Hospital, Southampton, Hampshire, UK. ${ }^{6}$ Department of Orthopedic Surgery, Sensory and Motor System Medicine, Graduate School of Medicine, University of Tokyo, Tokyo, Japan.

Received: 14 June 2019 Accepted: 9 December 2019

Published online: 26 December 2019

\section{References}

1. Wang YXJ, Kaplar Z, Deng M, Leung JCS. Lumbar degenerative spondylolisthesis epidemiology: a systematic review with a focus on gender-specific and age-specific prevalence. J Orthop Transl. 2016;11:39-52.

2. Denard PJ, Holton KF, Miller J, Fink HA, Kado DM, Marshall LM. Osteoporotic fractures in men (MrOS) study group. Back pain, neurogenic symptoms, and physical function in relation to spondylolisthesis among elderly men. Spine J. 2010;10:865-73.

3. Koreckij TD, Fischgrund JS. Degenerative spondylolisthesis. J Spinal Disord Tech. 2015;28:236-41.

4. Matz PG, Meagher RJ, Lamer T, Tontz WL Jr, Annaswamy TM, Cassidy RC, et al. Guideline summary review: an evidence-based clinical guideline for the diagnosis and treatment of degenerative lumbar spondylolisthesis. Spine J. 2016:16:439-48.

5. Steiger F, Becker HJ, Standaert CJ, Balague F, Vader JP, Porchet F, et al. Surgery in lumbar degenerative spondylolisthesis: indications, outcomes and complications. A systematic review. Eur Spine J. 2014;23:945-97.

6. Weinstein JN, Lurie JD, Tosteson TD, Hanscom B, Tosteson AN, Blood EA, et al. Surgical versus nonsurgical treatment for lumbar degenerative spondylolisthesis. N Engl J Med. 2007;356:2257-70.

7. Weinstein JN, Lurie JD, Tosteson TD, Zhao W, Blood EA, Tosteson AN, et al. Surgical compared with non-operative treatment for lumbar degenerative spondylolisthesis. Four-year results in the spine patient outcomes research trial (SPORT) randomized and observational cohorts. J Bone Joint Surg Am. 2009:9:1295-304.

8. DeVine JG, Schenk-Kisser M, Skelly AC. Risk factors for degenerative spondylolisthesis: a systematic review. Evid Based Spine Care. 2012;3:25-34.

9. Tallarico RA, Madom IA, Palumbo MA. Spondylolysis and spondylolisthesis in the athlete. Sports Med Arthrosc. 2008;16:32-8.

10. Toueg CW, Mac-Thiong JM, Grimard G, Parent S, Poitras B, Labelle H. Prevalence of spondylolisthesis in a population of gymnasts. Stud Health Technol Inform. 2010;158:132-7.

11. Jackson DW, Wiltse LL, Cirincione RJ. Spondylolysis in the female gymnast. Clin Orthop. 1976;117:68-73.

12. Rossi F. Spondylolysis, spondylolisthesis and sports. J Sports Med Phys Fitness. 1988;18:317-40.

13. Congeni J, McCulloch J, Swanson K. Lumbar spondylolysis: a study of natural progression in athletes. Am J Sports Med. 1997;25:248-53.

14. Chen J-C, Chan WP, Katz WP, Christiani DC. Occupational and personal factors associated with acquired lumbar spondylolisthesis of urban taxi drivers. Occup Environ Med. 2004;61:992-8.

15. Riihimäki $H$, Wickström $G$, Hänninen $K$, Luopajärvi T. Predictors of sciatic pain among concrete reinforcement workers and house painters--a five-year follow-up. Scand J Work Environ Health. 1989;15:415-23.

16. Yang S, Kim W, Choi KH, Yi YG. Influence of occupation on lumbar spine degeneration in men: the Korean National Health and nutrition examination survey 2010-2013. Int Arch Occup Environ Health. 2016;89:1321-8.

17. Jang TW, Ahn YS, Byun J, Lee JI, Kim KH, Kim Y, et al. Lumbar intervertebral disc degeneration and related factors in Korean firefighters. BMJ Open. 2016;6:e011587.

18. Froom P, Froom J, Van Dyk D, Caine Y, Ribak J, Margaliot S, et al. Lytic spondylolisthesis in helicopter pilots. Aviat Space Environ Med. 1984;55(6): $556-7$.

19. Mariconda M, Galasso O, Imbimbo L, Lotti G, Milano C. Relationship between alterations of the lumbar spine, visualized with magnetic resonance imaging, and occupational variables. Eur Spine J. 2007;16:255-66.

20. Yoshimura N, Muraki S, Oka H, Kawaguchi H, Nakamura K, Akune T. Cohort profile: research on osteoarthritis/osteoporosis against disability study. Int J Epidemiol. 2010;39:988-95.
21. Ishimoto Y, Yoshimura N, Muraki S, Yamada H, Nagata K, Hashizume H, et al. Prevalence of symptomatic lumbar spinal stenosis and its association with physical performance in a population-based cohort in Japan: the Wakayama spine study. Osteoarthr Cartil. 2012;20:1103-8.

22. Vogt MT, Rubin D, Valentin RS, Palermo L, Donaldson WF 3rd, Nevitt M, et al. Lumbar olisthesis and lower back symptoms in elderly white women. The Study of Osteoporotic Fractures. Spine. 1998;23:2640-7.

23. Denard PJ, Holton KF, Miller J, Fink HA, Kado DM, Yoo JU, et al. Lumbar spondylolisthesis among elderly men: prevalence, correlates, and progression. Spine. 2010;35:1072-8. https://doi.org/10.1097/BRS. Ob013e3181bd9e19.

24. He L-C, Wang Y, Gong J-S, Griffith JF, Zeng X-J, Kwok AWL, et al. Prevalence and risk factors of lumbar spondylolisthesis in elderly Chinese men and women. Eur Radio. 2014;21:441-8.

25. Lawrence JS. Rheumatism in coal miners. III. Occupational factors. Br J Ind Med. 1955;12:249-61.

26. Muraki S, Akune T, Oka H, Mabuchi A, En-Yo Y, Yoshida M, et al. Association of occupational activity with radiographic knee osteoarthritis and lumbar spondylosis in elderly patients of population-based cohorts: a large-scale population-based study. Arthritis Rheum. 2009;61:779-86.

27. Mahbub H, Laskar S, Seikh F, Altaf MH, Inoue M, Yokoyama K, et al. Prevalence of cervical Spondylosis and musculoskeletal symptoms among coolies in a City of Bangladesh prevalence of cervical Spondylosis and musculoskeletal symptoms among coolies in a City of Bangladesh. J Occup Health. 2006;48:69-73.

28. Videman T, Battié MC, Parent E, Gibbons LE, Vainio P, Kaprio J. Progression and determinants of quantitative magnetic resonance imaging measures of lumbar disc degeneration: a five-year follow-up of adult male monozygotic twins. Spine. 2008:33:1484-90.

29. Hung Y-J, Shih T, Chen B, Hwang Y-H, Ma L-P, Huang W-C, et al. The doseresponse relationship between cumulative lifting load and lumbar disk degeneration based on magnetic resonance imaging findings. Phys Ther. 2014;94:1582-93.

30. Ishihara $\mathrm{H}$, Tsuji $\mathrm{H}$, Hirano $\mathrm{N}$, Ohshima $\mathrm{H}$, Terahata N. Effects of continuous quantitative vibration on rheologic and biological behaviors of the intervertebral disc. Spine. 1992;17(3 Suppl):S7-12.

31. Vallone M, Bono F, Quendler E, Febo P, Catania P. Risk exposure to vibration and noise in the use of agricultural track-laying tractors. Ann Agric Environ Med. 2016;23:591-7.

32. Jensen A, Jepsen JR. Vibration on board and health effects. Int Marit Health. 2014;65:58-60.

33. Jacobsen S, Sonne-Holm S, Rovsing H, Monrad H, Gebuhr P. Degenerative lumbar spondylolisthesis: an epidemiological perspective. Spine. 2007;32: $120-5$.

\section{Publisher's Note}

Springer Nature remains neutral with regard to jurisdictional claims in published maps and institutional affiliations.

Ready to submit your research? Choose BMC and benefit from:

- fast, convenient online submission

- thorough peer review by experienced researchers in your field

- rapid publication on acceptance

- support for research data, including large and complex data types

- gold Open Access which fosters wider collaboration and increased citations

- maximum visibility for your research: over $100 \mathrm{M}$ website views per year

At BMC, research is always in progress.

Learn more biomedcentral.com/submissions 